\title{
Four frameshift mutations in neurofibromatosis type 1 caused by small insertions
}

\author{
Steven D Colman, Corinne R Abernathy, Vu T Ho, Margaret R Wallace
}

\begin{abstract}
We have been using heteroduplex analysis to assay individual exons within the NF1 gene in an effort to identify disease causing constitutional mutations in neurofibromatosis type 1 patients. Here we report the identification and characterisation of four insertional NF1 frameshift mutations in an analysis of exons 28-39 in a set of 78 patients. These include three 1 base pair insertions and one 2 base pair insertion. Three of these mutations can be attributed to replication slippage errors, while the mechanism behind the fourth may be related to formation of secondary structure during replication. It may be of significance that a majority of the previously reported small insertions in NF1 also lie within exons 28-39. $(\mathcal{H}$ Med Genet 1997;34:579-581)
\end{abstract}

Keywords: neurofibromatosis 1 ; insertions; mutation; ALL

\section{Department of \\ Pediatrics, Division of Genetics, University of Florida College of Medicine, Gainesville, FL, USA \\ S D Colman \\ C R Abernathy \\ V T Ho \\ M R Wallace}

Center for Mammalian Genetics, University of Florida College of Medicine, Gainesville, FL, USA

$S$ D Colman

M R Wallace

Departments of Biochemistry and Molecular Biology, and

Pathology and Immunology and Laboratory Medicine, University of Florida College of Medicine, Gainesville, FL, USA

M R Wallace

Correspondence to: Dr Wallace, Pediatric Genetics, Box 100296, University of Florida, Gainesville, FL 32610-0296, USA.

Received 29 October 1996 Revised version accepted for publication 29 January 1997
Neurofibromatosis type 1 (NF1), an autosomal dominant disease with a prevalence estimated at 1 in 3000 , is characterised by café au lait spots (CAL), cutaneous neurofibromas, axillary freckling, and Lisch nodules (hamartomas) of the iris. ${ }^{1}$ The NF1 gene, which is approximately $350 \mathrm{~kb}$ in length, comprises 59 exons. ${ }^{2}$ The large gene size and the fact that the majority of patients appear to have unique mutations has made the search for NF1 mutations difficult. The most recent distribution of the NF1 Genetic Analysis Consortium newsletter lists 194 characterised mutations, of which $21(11 \%)$ are small insertions. These mutations do not cluster at a single site. Only two of these insertions have occurred in unrelated patients; $62 \%$ of those reported do, however, fall within exons 28-39.

\section{Materials and methods}

PATIENT SAMPLES

Seventy eight unrelated NF1 patients (all meeting accepted diagnostic criteria) were studied. All patient samples were obtained under IRB approval with the patient's consent. Patient 1 is a white female with typical NF1 features and acute lymphoblastic leukaemia (ALL). Her mother also has NF1 but is not reported to have any signs of ALL or any other cancer. No other family information is available. Patient 2 is a white female who is a new mutation patient with multiple neurofibromas, including plexiform neurofibromas, and pseudarthrosis of the tibia which necessitated amputation at the age of 9 years. Patient 3 is a new mutation white male with CAL, plexiform orbit neurofibroma, multiple cutaneous neurofibromas, axillary freckling, optic glioma, high blood pressure, learning disabilities, hypotelorism, and bitemporal narrowing. $\mathrm{Pa}$ tient 4 is a new mutation white female with CAL, multiple neurofibromas, axillary freckling, macrocephaly, and learning disabilities.

HETERODUPLEX AND SEQUENCE ANALYSES

DNA purification and heteroduplex and sequence analyses of samples from each patient were conducted as previously described. ${ }^{3}$ In brief, DNA was purified from peripheral blood samples. Next, intron based oligonucleotide pairs were used to PCR amplify $12 \mathrm{NF} 1$ exons (exons 28-39, primers listed in Abernathy et $\left.a l^{4}\right)$. Amplified fragments were heated to $95^{\circ} \mathrm{C}$, cooled on ice, and incubated at room temperature to drive heteroduplex formation. Reannealed fragments were subsequently analysed by native polyacrylamide gel electrophoresis.

The relative levels of transcripts from each NF1 allele in patient 2's white blood cells were determined by RT-PCR and allele specific oligonucleotide (ASO) analysis via slot blots, as previously described. ${ }^{5}$ The ASO oligonucleotide specific to the normal sequence was $5^{\prime}$ TTTATTATGCTTCGGAA 3' and the oligonucleotide specific to the mutant sequence was 5' TTTATTATAAGCTTCGG 3'. Signals obtained in the ASO assay were quantified on a densitometer (Millipore BioImager).

\section{Results and discussion}

Four frameshift insertions were detected and characterised. (1) Patient 4 has an A inserted into exon 37 (6791 insA, where 6791 is the base in the cDNA sequence just before the insertion, 1 being the first base in the translation start site). This same mutation has been recently reported in an unrelated patient by Upadhyaya et al, ${ }^{6}$ who postulate that this region of exon 37 may be hypermutable. There is no obvious similarity in phenotype between these two patients. (2) Patient 3 has a $\mathrm{C}$ insertion in exon 36 (6709insC). (3) Patient 2 has an AA dinucleotide insertion in exon 29 (5289insAA), which was secondarily confirmed by restriction analysis based on the formation of a de novo AluI site (data not shown). (4) Patient 1 has an $\mathrm{A}$ insertion in exon 28 (4873ins A). The heteroduplex pattern for each of these mutations is shown in fig 1 . These mutations are all predicted to result in truncated neurofibromin. 

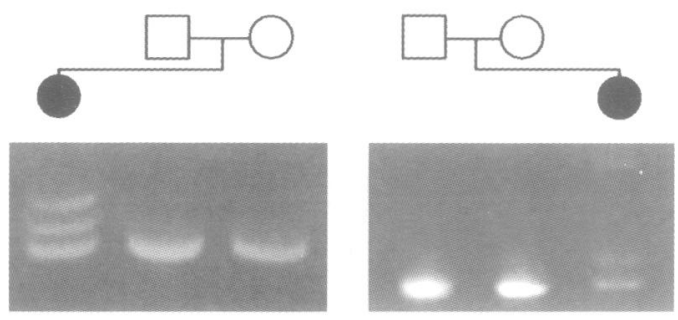

C
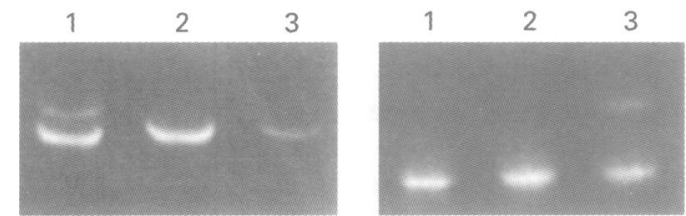

Figure 1 NF1 mutations identified by heteroduplex analysis. (A) This aberrant pattern was caused by a single bp insertion in exon 37 in DNA from patient 4 . (B) This pattern corresponded to a two base insertion in exon 29 from patient 2 . For $(A)$ and $(B)$, heteroduplexed DNA from unaffected parents are shown in adjacent lanes, as indicated by the pedigree. (C) The extra band in lane 1 is the result of a $1 \mathrm{bp}$ insertion in exon 36 of patient 3. Lanes 2 and 3 contain samples from NF1 patients apparently unaffected at this locus. (D) The extra band in lane 3 is caused by a 1 bp insertion in exon 28 of patient 1 . Lanes 1 and 2 contain samples from NF1 patients who are apparently unaffected at this locus.

In general, small insertions occur far less frequently than small deletions or single base changes. ${ }^{7}$ In accordance, small insertions in NF1 have been less frequently reported than small deletions and substitutions; small insertions reported to the NNFF consortium account for 21 of $194 \mathrm{NF} 1$ mutations (11\%), while small deletions and substitutions respectively account for $19 \%$ and $34 \%$ of those reported. We detected four small insertions in 78 DNA samples after analysing exons $28-39$. Our continued analysis of 66 of these patients has detected several deletions and substitutions scattered throughout the NF1 gene, but we have found no additional insertions. ${ }^{4}$ Thirteen of 21 small insertions previously reported to the NNFF consortium are also located within exons $28-39$. Therefore, with the inclusion of our report, 17 of 25 (68\%) small insertions detected lie within exons $28-39$. It cannot be determined at this time whether this putative "clustering" is significant or merely an artefact owing to bias of ascertainment. As the NF1 research community continues to identify insertions in NF1, this relationship will become clear.

Patient 1 is the second NF1 patient we have encountered who has both a frameshift mutation in exon 28 and ALL. ${ }^{5}$ NF1 patients are known to be at increased risk for leukaemia (particularly juvenile chronic myelogenous leukaemia and ALL to a lesser degree $^{8}$ ); however, mutations have not been reported for any other NF1 patients with ALL. Thus, the significance of our finding is unclear. The relevance of this association is further complicated by the fact that, although patient 1's mother also has NF1, she has not developed ALL. It may be of note that exon 28 is the first NF1 exon upstream from the two lymphoid

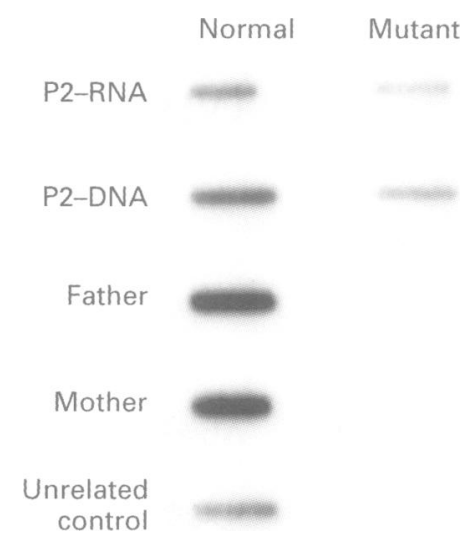

Figure 2 ASO analysis of mutant and normal exon 29 PCR products. The column on the left contains exon 29 PCR samples probed with an oligonucleotide corresponding to the normal exon 29 sequence. The duplicate samples on the right were probed with an oligonucleotide containing the 2 bp $A A$ mutation within the exon 29 sequence. P2-RNA refers to NF1 cDNA samples reverse transcribed from patient 2's total lymphocyte RNA and subsequently amplified by PCR. The remainder of the samples are PCR products amplified from genomic DNA. Densitometric quantification of these signals determined that there is no significant reduction of the steady state level of the message from the mutant allele (after normalisation of the relative signal from the normal $v$ the mutant oligonucleotide probe).

specific genes (EVI2A and EVI2B) transcribed from the opposite strand of intron $27 \mathrm{~b}$. The function of these genes is not known, but it is conceivable that there is some relationship between NF1 mutations in exon 28 and regulation of these genes.

Three of these mutations (the single base insertions) could be attributed to slippage during replication. In each case, the extra base is inserted next to an identical base (that is, insertion of an A next to an A). The independent recurrence of 6791 insA suggests that there might be a driving force behind insertions at specific sites. Insertions can also be mediated by secondary structure. The two base AA insertion seen in exon 29 of patient 2 occurs in the middle of a $40 \mathrm{bp}$ stretch that is $75 \% \mathrm{~A}+\mathrm{T}$. This sequence could provide an opportunity for secondary structure formation and subsequent mispairing during replication.

It has been suggested that NF1 mutations, like frameshift mutations in many other genes, can result in reduced levels of mutant transcripts relative to normal ones. ${ }^{59-11}$ However, there have also been reports of small mutations which do not have this effect. ${ }^{312}$ ASO analysis of leucocyte RNA from patient 2 detected approximately equal levels of message from both the normal and mutant NF1 alleles (fig 2), thus reinforcing the concept that the effect of small frameshift insertions on transcript levels is variable in NF1.

We thank Drs Francis Collins, Charles Williams, Roberto Zori and Ms Heather Stalker for helping in obtaining samples, and Ms Reena Kamath for technical assistance. The NF1 InterMs Reena Kamath for national Genetic Analysis Consortium data are provided by the National Neurofibromatosis Foundation, through its newsletter editor Dr Bruce Korf. This work was funded by grants to MRW from the Hayward Foundation, the National Neurofibromatosis Foundation, the American Cancer Society (Florida Division), and the Children's Miracle Network. Further support included NIH funding to MRW (R29 NS31550) and SDC (T32 CA09126, F32CA61639), and the Raymond C Philips Research and Education Contract, Children's Medical Services, from the Department of Health and Rehabilitative Services, State of Florida. 
1 Riccardi VM. Neurofibromatosis. Phenotype, natural history and pathogenesis. 2nd ed. Baltimore: The Johns Hopkins and pathogenesis. 2nd
University Press, 1992.

$2 \mathrm{Li} \mathrm{Y,} \mathrm{O'Connell} \mathrm{P,} \mathrm{Huntsman-Breidenbach} \mathrm{HH}$, et al. $\mathrm{Li} Y$, O'Connell P, Huntsman-Breidenbach $\mathrm{HH}$, et al. Genomic organization of the
$(N F 1)$. Genomics 1995;25:9-18.

3 Abernathy CR, Colman SD, Kousseff BG, Wallace MR. Two NF1 mutations: frameshift in the GAP-related domain, and loss of two codons toward the 3' end of the gene. Hum Mutat 1994;3:347-52.

4 Abernathy CR, Rasmussen SA, Stalker HJ, et al. NF1 mutation analysis using a combined heteroduplex/SSCP approach. Hum Mutat (in press).

5 Colman SD, Collins FS, Wallace MR. Characterization of a single-base pair deletion in neurofibromatosis type 1. Hum Mol Genet 1993;2:1709-11.

6 Upadhyaya M, Osborn M, Maynard J, Harper P. Characterization of six mutations in exon 37 of neurofibromatosis type 1 gene. Am $\mathcal{F}$ Med Genet 1996;67:421-3.
7 Cooper DN, Krawacz M. Human gene mutation. Oxford: BIOS Scientific Publishers, 1993.

8 Stiller CA, Chessells JM, Fitchett M. Neurofibromatosis and childhood leukaemia/lymphoma: a population-based UKCCSG study. Br f Cancer 1994;70:969-72.

9 Ainsworth P, Rodenhiser D, Stuart A, Jung J. Characterization of an intron 31 splice junction mutation in the neurofibromatosis (NF1) gene. Hum Mol Genet 1994;3:1 179-81.

10 Hoffmeyer S, Assum G, Griesser J, Kaufmann D, Nurnberg $P$, Krone W. On unequal allelic expression of the neurofibromin gene in neurofibromatosis type 1 . Hum $\mathrm{Mol}$ Genet 1995;4:1267-72.

11 Legius E, Hall BK, Wallace MR, Collins FS, Glover TW Ten base pair duplication in exon 38 of the NF1 gene. Hum Mol Genet 1994;3:829-30.

12 Stark M, Assum G, Kaufmann D, Keher H, Krone W. Analysis of segregation and expression of an identified mutation at the neurofibromatosis type 1 locus. Hum Genet 1992;90:356-9. 